\title{
EFFECT OF QUERCETIN ON FILIFORM AND FUNGIFORM PAPILLAE OF THE TONGUE OF ALBINO RATS WITH INDUCED DIABETES
}

\author{
Nesma Mohamed Khalil* and Dina Ali Nagui***
}

\begin{abstract}
Objective: The aim of the present work was to study the effect of Quercetin_on the structure of filiform and fungiform papillae of the tongue in albino rats with streptozotocin induced diabetes.

Design: Thirty adult male Wistar rats were divided into 3 equal groups (10 rats each): Group 1 (control group), Group 2: (diabetic group) and Group 3: (Quercetin treated diabetic group). In both groups $2 \& 3$ diabetes was induced by single intraperitoneal injection of streptozotocin $(50 \mathrm{mg} / \mathrm{kg})$. After confirmation of diabetes, Quercetin was administrated to group $3(50 \mathrm{mg} / \mathrm{kg} / \mathrm{day})$ orally for 12 weeks. Then all rats were sacrificed and the tongues were dissected out for light microscopic (LM) examination, immunohistochemical examination, histomorphometric analysis and scanning electron microscopic (SEM) examination.
\end{abstract}

Results: LM examination of diabetic group revealed disturbances in filiform papillae with hyperkeratosis. Fungiform papillae exhibited slight elongation with separation between taste bud cells. SEM examination revealed disturbance in the normal orientation of filiform papillae which appeared thin. On the other hand, in Quercetin treated group, there was marked improvement in the shape and orientation of the papillae. However, some changes were observed by LM which included hyperkeratosis and in SEM some filiform papillae appeared thin. Immunohistochemical analysis revealed strong reaction for caspase 3 in diabetic group, while in Quercetin treated group the reaction was mild. Quercetin treatment resulted in significant decrease in both area percentage of immunopositive cells and the optical density of caspase 3 immunostaining which was significantly increased after induction of diabetes.

Conclusions: In a rat model, Quercetin can to a great extent overcome most of the detrimental effects of diabetes on the structure of both filiform and fungiform papillae of the tongue.

KEY WORDS: Quercetin, caspase 3, diabetes, papillae

\footnotetext{
* Department of Oral Biology, Faculty of Dentistry, Alexandria University, Egypt
} 


\section{INTRODUCTION}

Diabetes mellitus (DM) is a heterogeneous group of metabolic disorders manifested by abnormally high glucose level in the blood. The hyperglycemia results from a deficiency in insulin secretion caused by pancreatic $\beta$-cell dysfunction or from resistance of the peripheral receptors to the action of insulin in liver and muscle, or a combination of both. Chronic hyperglycemia leads to long-term damage to many organs including the heart, eyes, kidneys, nerves, and vascular system. ${ }^{(1)}$

The number of people suffering from diabetes is increasing due to population growth, aging, urbanization, and increasing prevalence of obesity and physical inactivity. ${ }^{(2)}$ It is estimated that the global number of adults affected by diabetes will reach 439 million in 2030, most of them are type 2 DM..$^{(3,4)}$

Diabetes has many complications which include the development of diabetes-related pathology in the retina, kidney, and peripheral nerves. It also results in accelerated atherosclerosis affecting arteries that supply the brain, heart, and lower extremities. ${ }^{(5)}$ Both Diabetes and impaired glucose tolerance lead to increased cardiovascular disease risk three to eight-folds. ${ }^{(6)}$ New blood vessels formation due to ischemia is impaired in diabetic patients, resulting in decreased collateral vessels formation in ischemic hearts, and in non-healing foot ulcers. ${ }^{(7)}$

Moreover, diabetes is considered as a risk factor for a number of oral disorders including: gingivitis and periodontitis, dental caries, oral infections such as candidiasis, taste disorders, and oral mucosal diseases. DM has been also attributed to salivary dysfunction leading to diminished salivary flow and subsequent oral and tongue burning sensation and concomitant enlargement of the parotid salivary glands. ${ }^{(8)}$

Taste alteration may be experienced by diabetic patients even before development of the other well-known complications. ${ }^{(9)}$ Unfortunately, taste disorders are commonly overlooked because they are not considered critical to life. However, decreased taste can affect the appetite which results in weight loss, poor nutrition, low immunity, and deterioration in medical health. ${ }^{(10)}$

Free radicals are reactive molecules that possess unpaired electrons and to be stable and gain electrons, they have to oxidize other molecules. This reaction leads to formation of other free radicals. These radicals can oxidize important cellular components like deoxyribonucleic acid (DNA), carbohydrates and proteins leading to damage. ${ }^{(11)(12)}$ These radicals are termed reactive oxygen species (ROS) and include superoxide (O2-), hydrogen peroxide (H2O2), and hydroxyl.(13) ROS are formed inside the cell from mitochondrial oxidative phosphorylation or from environmental pollutants like smoking, radiation and pesticides. (12)\&(13) Antioxidant enzymes in our body protect us against free radicals. However, when ROS overwhelm the antioxidant enzymes, this results in a state known as oxidative stress. ${ }^{(14)}$ It is believed that most of the diabetic complications are related to increase in oxidative stress in diabetic patients. ${ }^{(15,16)}$

Flavonoids are a group of naturally occurring polyphenolic compounds found in photosynthesising cells. They are present in high doses in many antioxidant supplements and herb-containing medicaments. ${ }^{(17)}$ Quercetin (3,3', 4', 5,7-pentahydroxyflavone) is one of the most commonly used flavonols in human diet, as it is generally distributed in different types of fruits, tea, pepper, coriander, fennel, radish and berries. ${ }^{(18)}$

Quercetin has been associated with the prevention and treatment of cardiovascular diseases and cancer due to its anti-inflammatory and antioxidant properties. Also, many studies have been performed using Quercetin for the management of DM. It was suggested that it exerts its antidiabetic action by reducing intestinal glucose absorption, decreasing lipid peroxidation, and enhancing the antioxidant enzymes activity in the body. ${ }^{(19-22)}$

Since most of the hypoglycemic drugs have unavoidable side effects and the cost of insulin 
therapy may be unsuitable for low income diabetic patients ${ }^{(23)}$, so most of the current efforts look for alternative more safe and natural compounds for the treatment of diabetes and its complications. So, the aim of the present work was to study the histological, ultrastructural and immunohistochemical effect of diabetes on the filiform and fungiform papillae of the tongue of albino rats and the possible therapeutic effect of the concurrent Quercetin administration.

\section{MATERIALS AND METHODS}

\section{Experimental procedures}

This study was approved by the ethical committee of Faculty of Dentistry Alexandria University. Thirty male Wistar rats about 5 months old weighing from 220-250 grams were used in this study. They were kept in the Experimental Animal House, Faculty of Agriculture, Alexandria University. The animals were maintained in metal cages, five rats per cage at $24 \pm 1^{\circ} \mathrm{C}$ and 12 hours light/dark cycles. Rats were fed a standardized diet with water ad libitum. After 10 days of acclimatization, animals were randomly allocated into 3 groups, each consists of 10 rats: Group 1 (control group), Group 2: (diabetic group) and Group 3: (Quercetin treated diabetic group).

In both groups $2 \& 3$ : diabetes was induced by a single intraperitoneal injection of streptozotocin (STZ, $50 \mathrm{mg} / \mathrm{kg}$ ). Confirmation of diabetes was done when a stable level of hyperglycemia was obtained (250-350 mg/dl). ${ }^{(24)}$

In group 3, after confirmation of diabetes (after 2 weeks of STZ injection), Quercetin (Sigma Aldrich chemical Company, St. Louis, USA) was administrated orally at a dose of $50 \mathrm{mg} / \mathrm{kg} /$ day. (24) It was supplied in powder form, dissolved in distilled water and administrated orally using oral gavage syringe for 12 weeks ${ }^{(25)}$ after confirmation of diabetes. Both groups $1 \& 2$ received orally $0.5 \mathrm{ml}$ of distilled water daily.

At the end of the experimental period, all rats were euthanized with a lethal dose $(150 \mathrm{mg} / \mathrm{kg}$ body weight) of sodium thiopental. Afterwards, tongues from all rats was dissected out and divided into two halves. The right halves of the tongues were prepared for light microscopic examination and immunohistochemical analysis and the left halves were prepared for scanning electron microscopic examination.

\section{Light microscopic examination}

Tongue specimens were fixed in $10 \%$ neutral buffered formalin, rinsed in distilled water and then dehydrated in ascending concentrations of alcohol. Specimens were then cleared in xylene and embedded in paraffin blocks. $5 \mu \mathrm{m}$ thick longitudinal sections were cut from each block and stained with haematoxylin and eosin stain. ${ }^{(26)}$

\section{Scanning electron microscopic examination}

The specimens were cleaned with soft brush under running water to remove mucous from the dorsal surface of the tongue. Then they were fixed in $2.5 \%$ glutaraldehyde in $0.1 \mathrm{M}$ phosphate buffer ( $\mathrm{pH}$ 7.2) for 1.5-2 hours. Afterwards, they were rinsed two times in phosphate buffer for 15 minutes each. Then specimens were post-fixed in osmium in $0.1 \mathrm{M}$ phosphate buffer $(\mathrm{pH}$ 7.2) for about 2 hours. Unbound traces of osmium were washed out with two rinses in buffer for 15 minutes each. Afterwards, specimens were dehydrated in ascending concentrations of ethanol (40-60-80-95$100 \%$ ), about 15 minutes for each one, then were dried by critical point drying. Finally, the specimens were coated using a sputter coater with a thin layer of gold and were examined on JOEL 5300 JSM scanning electron microscope at Faculty of Science, Alexandria University.(27)

\section{Immunohistochemical examination}

From each specimen, $5 \mu \mathrm{m}$ thick sections were dewaxed in xylene and rehydrated in graded alcohol series till distilled water. To retrieve antigen, sections were boiled in $0.01 \mathrm{~m}$ sodium citrate buffer $(\mathrm{pH}=6.0)$ in a microwave for about 20 minutes. 
Caspase 3 primary antibody (Thermo Fisher Scientific, Fremont, USA) was diluted at 1:100 and then incubated at $4^{\circ} \mathrm{C}$ overnight. Afterwards, the slides were rinsed 3 times in tris buffered saline (TBS) (10 minutes each) and then incubated with secondary antibody (Ultra Vision detection Kit, Dako Cytomation). The slides were rinsed again 3 times in TBS (10 minutes each). Afterwards, they were stained with 3, 3' diaminobenzidinetetrahydrochloride. Then the sections were counterstained with Mayer's haematoxyline. Negative control reaction was carried out without primary antibody. ${ }^{(28)}$

\section{Histomorphometric analysis}

From each specimen, 3 sections at different standardized depths were selected for analysis. From each section one photograph at magnification power (x400) was taken. In each photograph, quantitative analysis was done regarding two parameters: the area percentage of caspase 3 immunopositive cells and the optical density (OD) of the immunostaining. (29) Then the mean of the 3 sections of the same specimen was obtained.

The area percent and the color intensity of immune reaction was done using ImageJ $1.46 \mathrm{r}$ (National Institutes of Health, Bethesda, MD). The optical density was calculated according to the following equation:
$\mathrm{OD}=\mathrm{LOG}$ (maximum intensity/mean intensity), where maximum intensity is 255 . $^{(29)}$ In image analysis, the pixel intensity of any colour ranges from 0 to 255 . Zero represents the darkest shade and 255 represents the lightest shade of the colour. ${ }^{(30)}$

The same procedure was repeated in each of the 10 specimens in each group. The results were expressed by means and standard deviation. Comparison between groups was done using analysis of variance. The significance level was 0.05 and $p$ values less than 0.05 were considered significant. Statistical analysis was done using SPSS version 20 (IBM Corp, Armonk, NY).

\section{RESULTS}

\section{Histological Results:}

Light microscopic examination of the dorsal surface of the tongue of the control group 1 revealed normal structure and arrangement of the filiform and fungiform papillae. The filiform papillae appeared cone- shaped, covered by keratinized stratified squamous epithelium (Fig.1a). The fungiform papillae were seen with their characteristic mushroom-shape and with barrel-shaped taste buds located at the superior surface. (Fig.1b)

Examination of the diabetic group 2 showed marked alteration of the shape of the tongue papillae.

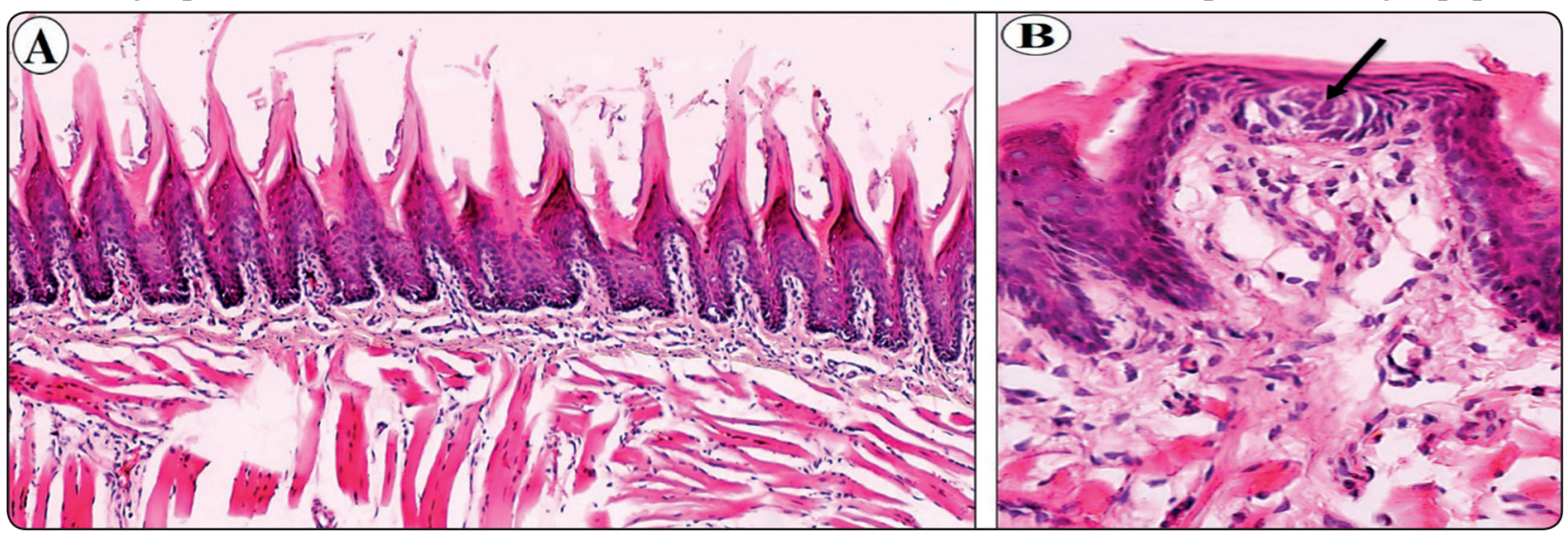

Fig. (1) (a\&b): Light micrograph (LM) of the filiform (a) \& fungiform papillae (b) of the dorsal surface of the tongue of the control group 1. A: Cone shaped filiform papillae covered by keratinized epithelium can be seen. B: Fungiform papilla is covered by a thin keratinized epithelium. A barrel shaped taste bud (arrow) can be seen on the superior surface of the papilla. (H\&E, A:100x, B:400x) 


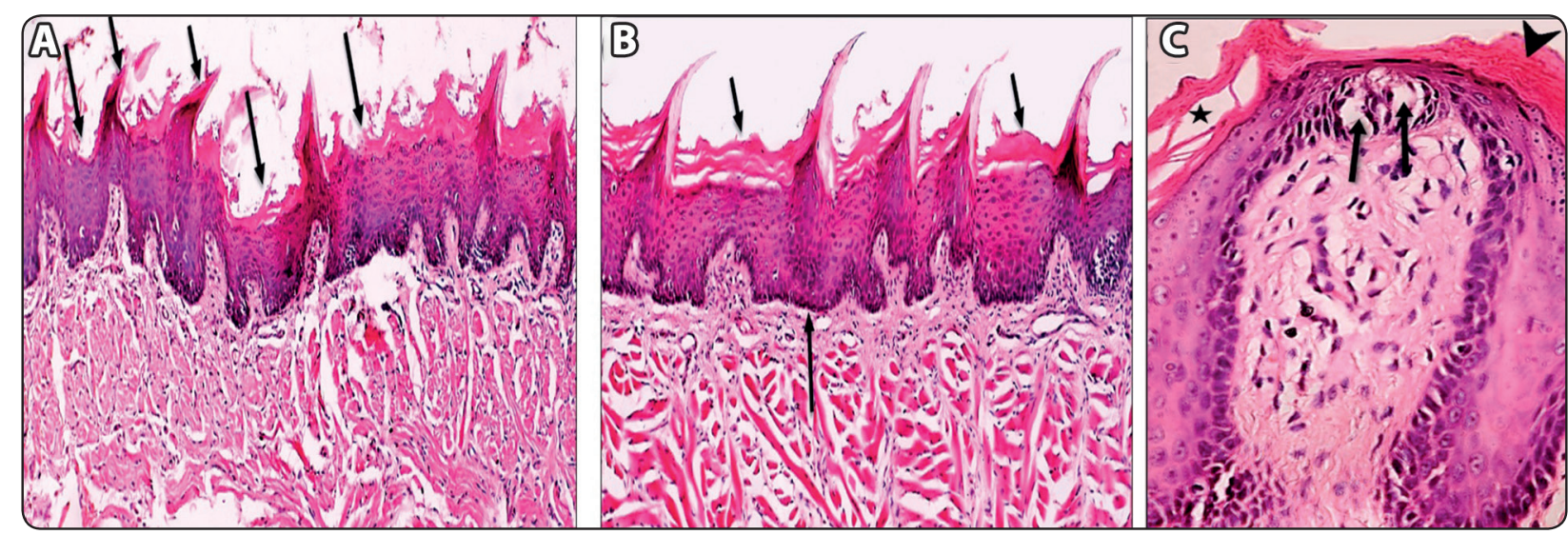

Fig. (2) (a-c): LM of the filiform (a\&b) \& fungiform papillae (c) of the dorsal surface of the tongue of the diabetic group 2. A: shows distortion of the normal organization of the filiform papillae. Some papillae appeared short (short arrows), while other areas revealed complete loss of the papillae (long arrows). B: shows areas in the dorsal surface of the tongue devoid of papillae with evident hyperkeratosis (short arrows). Loss of normal connective tissue papillae (long arrow) is also seen. (C): shows slight elongation of the fungiform papilla which is covered by a relatively thick keratin layer (arrow head) and other area shows splitting within the keratinous covering (star). The cells of the taste bud appear detached from each other (arrows). (H\&E, A\&B: 100x, C: 400x)

Some filiform papillae appeared shorter. Other areas showed loss of the characteristic flame shape of the papillae and appeared with hyperkeratosis. Loss of the normal connective tissue papillae was also observed (Fig.2a\&b). Moreover, fungiform papillae appeared slightly elongated. The covering keratin layer appeared slightly thickened with separation within its layers. Taste duds showed apparent separation between its cells (Fig.2c).

In the Quercetin-treated group 3, most of the filiform papillae appeared typically cone-shaped covered by a uniform layer of keratinized stratified squamous epithelium (Fig.3a). However, the normal thickness of epithelial covering of some papillae was improperly restored, instead an abnormally thickened keratinous layer was seen (Fig.3b). The histological structure of the fungiform papillae and apically-located taste buds was mostly restored (Fig.3 c\&d).

\section{Scanning electron microscopic results:}

Ultrastructural examination of the dorsal surface of the tongue of control group 1 showed normal anteroposterior arrangement of the filiform papillae along the surface of the tongue. They appeared as uniform conical projections with tapered apices. Fungiform papillae were detected in between the filiform ones with the taste pore clearly centered over their broad summit (Fig.4)

When the diabetic group 2 was examined, the lingual papillae revealed evident disorganization. The filiform papillae showed marked thinning along their length with obvious clefting within the keratinous tip or desquamation of the epithelial covering. There was discrete flattened and irregular interpapillary zones. In other areas, the fungiform papilla was reduced in size and had a masked taste pore (Fig.5).

Ultrastructural examination of the Quercetin group 3 showed almost normal shape and arrangement of most of the filiform papillae, which restored their uniform thickness and length. However, few papillae were still thin or had superior concave surface. Well defined fungiform papillae were seen between the filiform ones (Fig.6). 

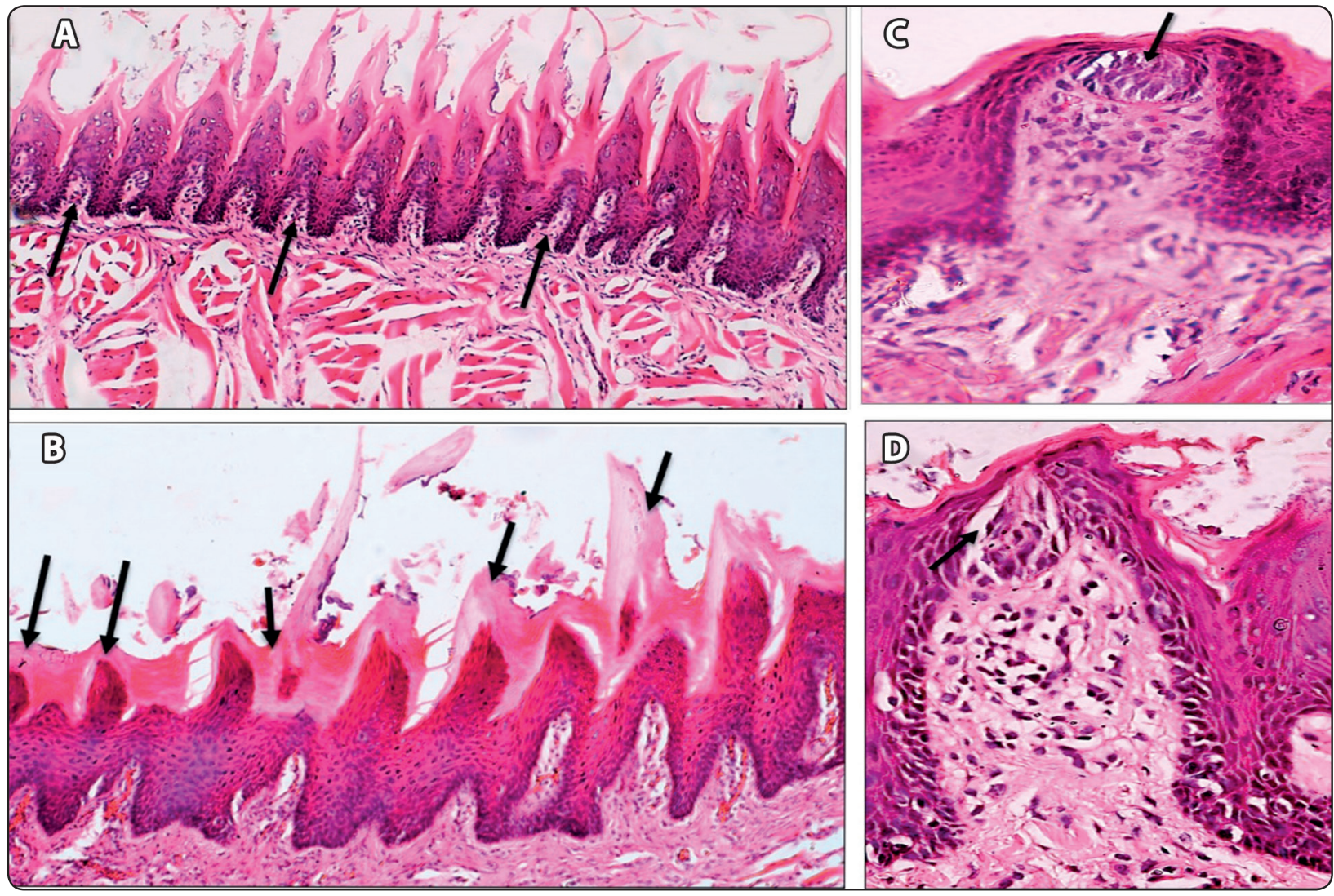

Fig.3 (a-d): LM of the filiform (a\&b) \& fungiform papillae (c\&d) of the dorsal surface of the tongue of the Quercetin treated group 3. (A): shows almost complete restoration of the characteristic shape of the filiform papillae with preserved connective tissue papillae (arrows). (B): Other areas showed some changes like hyperkeratosis (short arrows) and loss of the conical shape of the papillae (long arrows). (C): shows the mushroom shaped fungiform papilla with the barrel shaped taste bud (arrow). (D): shows slight separation between the taste cells and the periphery of the taste bud (arrow). (H\&E, A: 100x, B: 200x, C\&D: 400x)

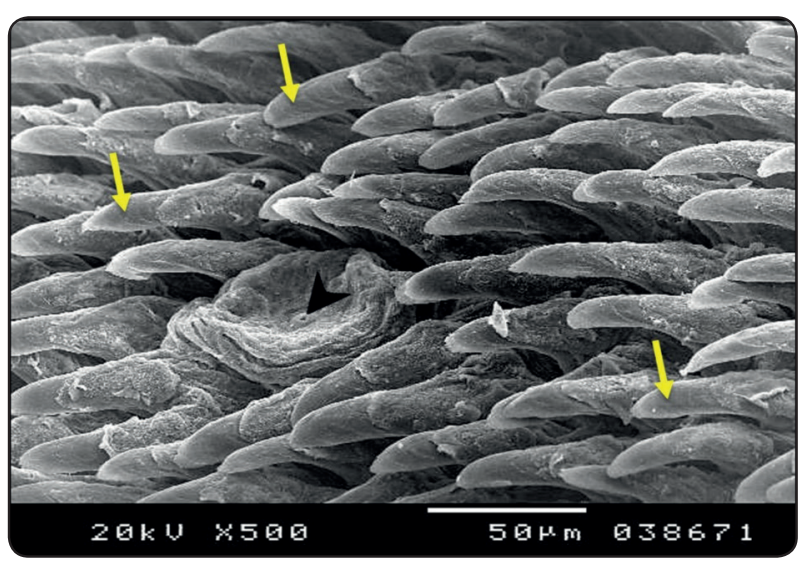

Fig. (4) Scanning electron micrograph (SEM) of the dorsal surface of the tongue of control group 1 showing regularly distributed filiform papillae (arrows) with tapered ends and uniform anteroposterior inclination. A fungiform papilla can be seen with a well-defined taste pore at its center (arrow head). (500x) 

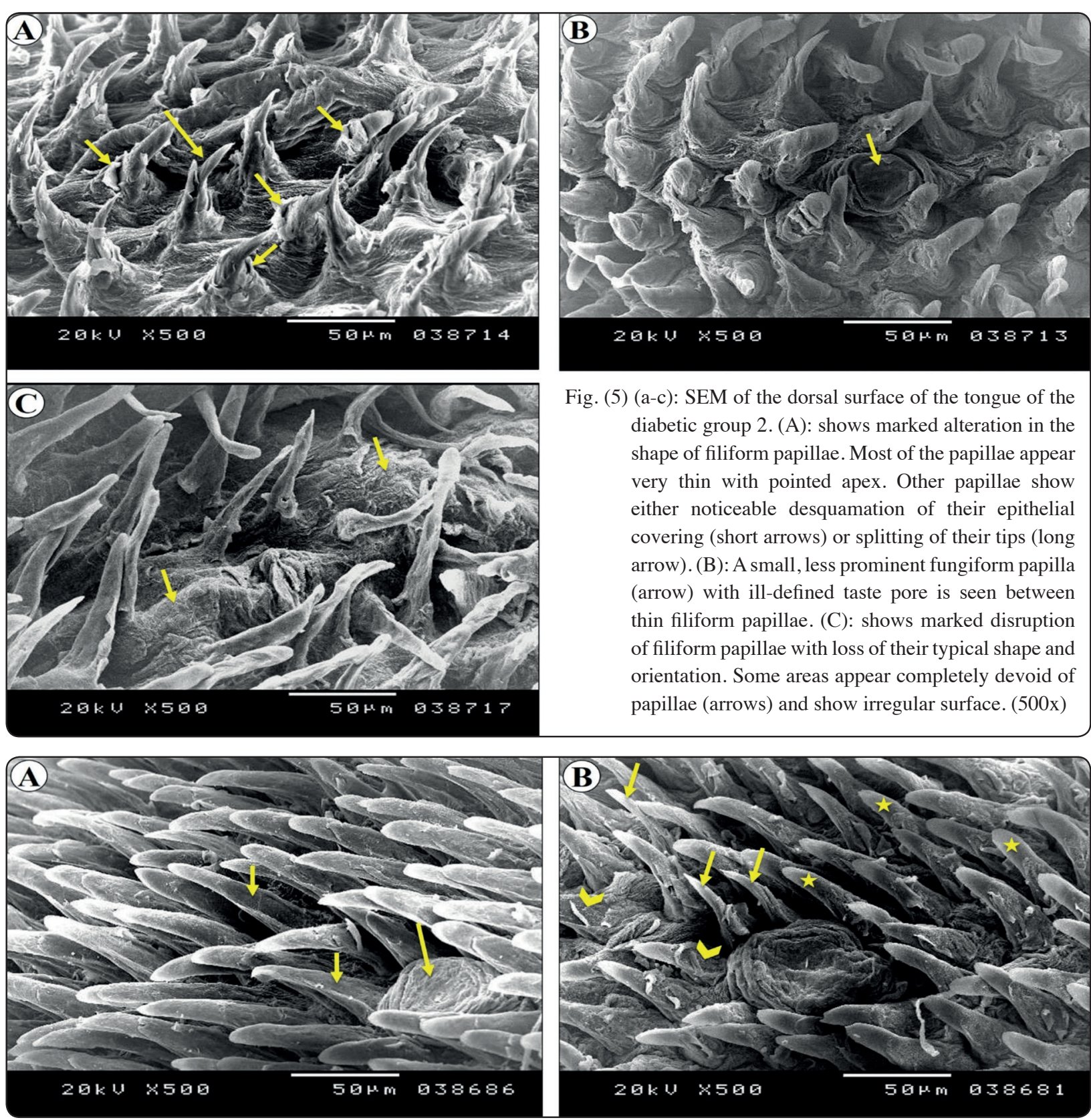

Fig. (6) (a\&b): SEM of the dorsal surface of the tongue of Quercetin treated group 3. (A): shows almost restoration of the typical shape of the filiform papillae. Some filiform papillae have concave superior surface (short arrows). A well-defined fungiform papilla (long arrow) can be seen in-between the filiform papillae. (B): Most of the filiform papillae appear with their typical cone shape (stars). Some filiform papillae appear very thin (arrows) or with eroded keratin layer (arrow heads). (500x)

\section{Immunohistochemical results}

Light microscopic examination of caspase 3 immuno-stained sections revealed very mild or almost no reaction in the tongue papillae in the control group 1 (Fig. 7). On the other hand, in

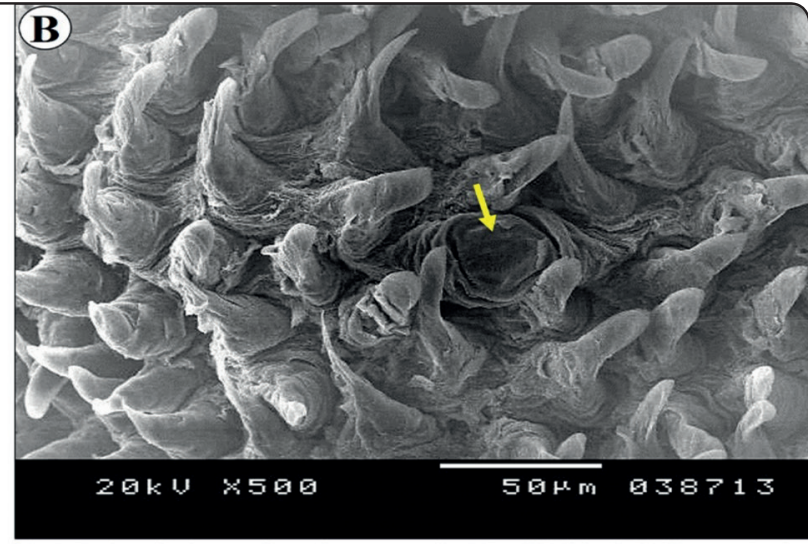

Fig. (5) (a-c): SEM of the dorsal surface of the tongue of the diabetic group 2. (A): shows marked alteration in the shape of filiform papillae. Most of the papillae appear very thin with pointed apex. Other papillae show either noticeable desquamation of their epithelial covering (short arrows) or splitting of their tips (long arrow).(B): A small, less prominent fungiform papilla (arrow) with ill-defined taste pore is seen between thin filiform papillae. (C): shows marked disruption of filiform papillae with loss of their typical shape and orientation. Some areas appear completely devoid of papillae (arrows) and show irregular surface. (500x) 


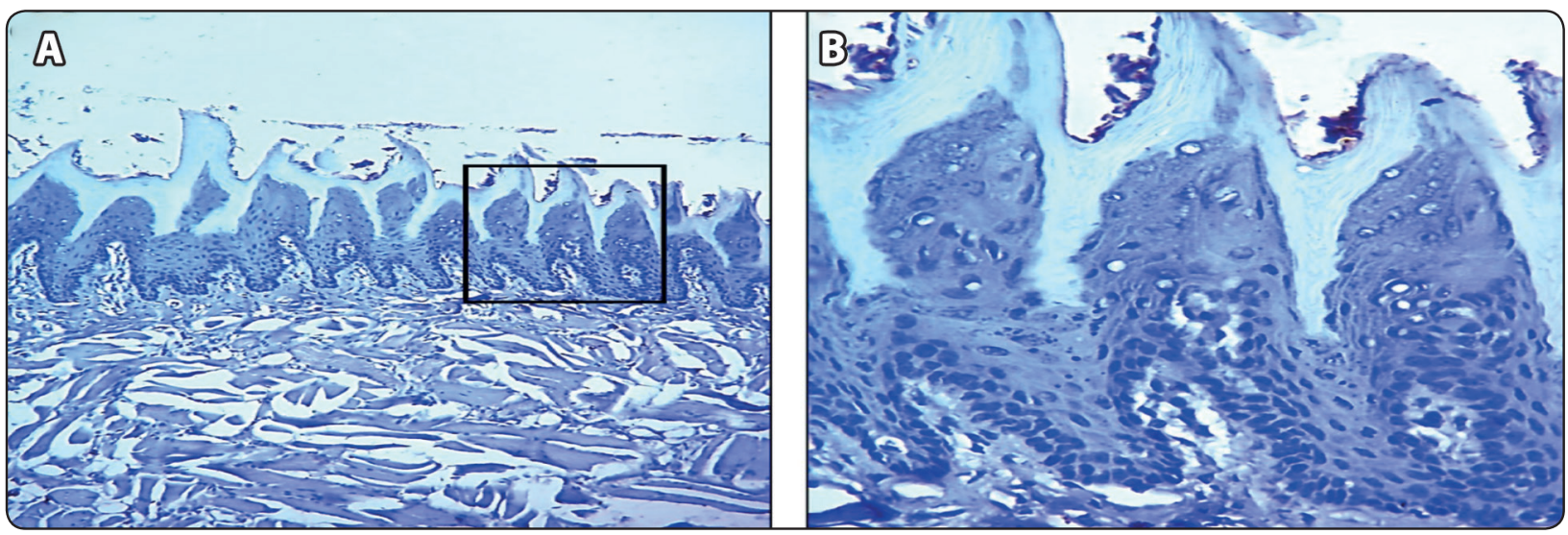

Fig. (7) (a\&b): LM of caspase 3 immunostaining of tongue papillae of control group 1 showing almost no immunostaining of the filiform papillae. (a 100x, b 400x)

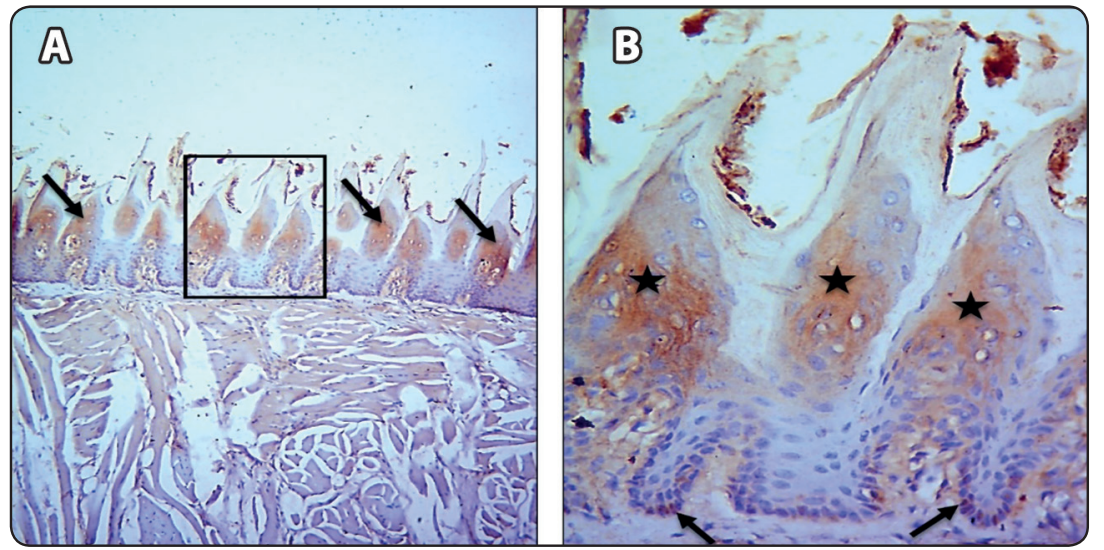

Fig. (8) (a\&b): LM of caspase 3 immunostaining of tongue papillae of diabetic group 2. (A): shows strong immuno-positivity of caspase 3 staining of tongue papillae (arrows). (B): Higher magnification of the inset in A showing the strong staining in the center of the papillae especially in the granular and prickle cells (black stars). Positively stained basal cells are also seen (arrows). (a 100x, b 400x)
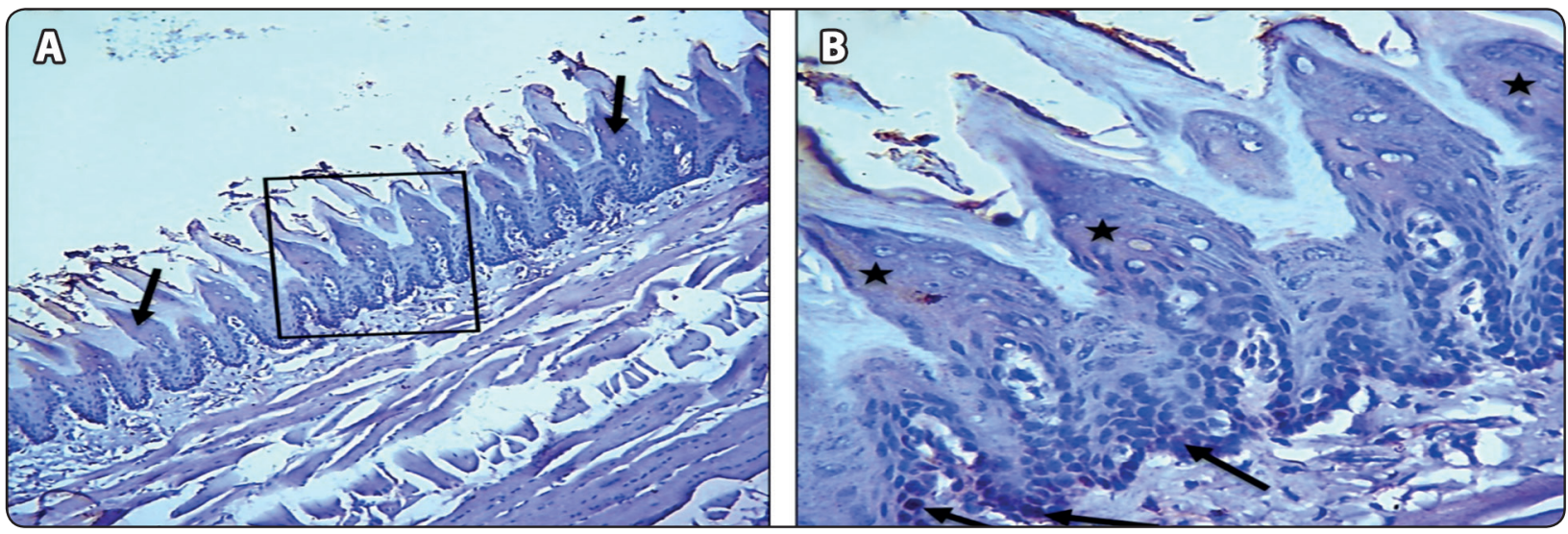

Fig. (9) (a\&b): LM of caspase 3 immunostaining of tongue papillae of Quercetin treated group 3. (A): showing mild staining of tongue papillae (arrows). (B): Higher magnification of the inset in A showing the mild immunostaining of some granular, prickle (stars) and basal cells (arrows). (a 100x, b 400x) 


\subsection{Histomorphometric results}

The area percentage of cells with positive reaction to caspase 3 showed significant increase in diabetic group 2 compared to control group 1 (21.2\% \& $3 \%$ respectively), where $\mathrm{p}_{1}<0.001$. After Quercetin treatment, there was a significant decrease in area percent of immunopositive cells compared to diabetic group2 $(10.5 \% \& 21.2 \%$ respectively, $\left.\mathrm{p}_{3}<0.001^{*}\right)$. However, the difference between the control group 1 \& Quercetin treated group 3 was significant $\mathrm{p}_{2}<0.001$.

The optical density of caspase 3 immunostaining in diabetic group 2 was significantly higher than control group1 ( $0.1 \& 0.021$ respectively, $\mathrm{p}_{1}=0.002$ ). On the other hand, Quercetin treatment resulted in significant decrease in the optical density that was very close to control group $1(0.029 \& 0.021$ respectively, $\mathrm{p}_{2}=0.722$ ).

TABLE (1): Comparison between the three studied groups regarding the area percentage of caspase 3 immunopositive cells and the optical density of the immunostaining

\begin{tabular}{|c|c|c|c|c|c|}
\hline & $\begin{array}{c}\text { Control group 1 } \\
(\mathbf{n}=\mathbf{1 0})\end{array}$ & $\begin{array}{c}\text { Diabetic group 2 } \\
(\mathbf{n = 1 0})\end{array}$ & $\begin{array}{c}\text { Quercetin group 3 } \\
(\mathbf{n = 1 0})\end{array}$ & F & p \\
\hline Area \% of cells & $3 \pm 0.8$ & $21.2 \pm 5.1$ & $10.5 \pm 3.9$ & $59.632^{*}$ & $<0.001^{*}$ \\
\hline Sig. bet. grps & \multicolumn{3}{|c|}{$\mathrm{p}_{1}<0.001^{*}, \mathrm{p}_{2}<0.001^{*}, \mathrm{p}_{3}<0.001^{*}$} & $11.920^{*}$ & $0.003^{*}$ \\
\hline Optical density & $0.021 \pm 0.012$ & $0.1 \pm 0.084$ & $0.029 \pm 0.025$ & & \\
\hline Sig. bet. grps & \multicolumn{3}{|c|}{$\mathrm{p}_{1}=0.002^{*}, \mathrm{p}_{2}=0.722, \mathrm{p}_{3}=0.005^{*}$} & & \\
\hline
\end{tabular}

F: F for ANOVA test, Pairwise comparison bet. each 2 groups was done using Post Hoc Test (Tukey)

H: H for Kruskal Wallis test, Pairwise comparison bet. each 2 groups was done using Post Hoc Test (Dunn's for multiple comparisons test)

p: $p$ value for comparing between the three studied groups

p1: p value for comparing between Control and Diabetic

p2: p value for comparing between Control and Quercetin

p3: p value for comparing between Diabetic and Quercetin

*: Statistically significant at $p \leq 0.05$ 


\section{DISCUSSION}

The universal escalation of diabetes in developed and developing countries poses a great health challenge. Both forms of diabetes $(1 \& 2)$ are associated with a great variety of microvascular complications such as retinopathy, cardiomyopathy, nephropathy, and neuropathy which result from alterations in lipid metabolism, increased hyperglycemia-mediated oxidative stress, endothelial cell dysfunction, and cellular apoptosis. ${ }^{(31)}$

Quercetin is a natural element of the flavonoid group that is mainly common as a secondary metabolite in plants. It was established that natural antioxidants are usually harmless to human body as they prevent early aging via blocking the detrimental effects of reactive oxygen species (ROS) on biological chain reactions. It was demonstrated that Quercetin has anticarcinogenic and antiinflammatory properties beside its antioxidant and free radical scavenging effects. It can decrease the oxidative injury to different tissues such as the brain and the heart in ischemic reperfusion damage. ${ }^{(32)}$

Therefore, in the present study, we investigated the possible therapeutic effect of quercetin on the degenerative changes affecting the tongue papillae as a result of induction of diabetes mellitus in rats. This was demonstrated histologically, ultrastructurally and immunohistochemically.

In this study, STZ was used to induce diabetes in rats. Lenzen ${ }^{(33)}$ and Suzuki et al ${ }^{(34)}$ stated that STZ induced diabetes is a well stablished animal model for investigation of different pharmacological agents. It can induce type I diabetes through rapid destruction of $\beta$-cells of pancreas. Alloxan is another pharmacological agent used for induction of diabetes, however, Islas-Andrade et al ${ }^{(35)}$ proved that STZ can induce a permanent and stable diabetic model compared to alloxan.

The light microscopic results of our study showed that diabetes resulted in marked alterations in the structure of both filiform and fungiform papillae. Scanning electron microscopic results also confirmed the LM results. Filiform appeared very thin with desquamation of their epithelial covering. Some fungiform papillae were less prominent and reduced in size. These results are in coincidence with those of Akiya et al ${ }^{(36)}$ who found that diabetes resulted in atrophy of lingual papillae and alterations in lingual mucosal capillaries. In addition, Mohsen et al ${ }^{(37)}$ and Ortug et al ${ }^{(38)}$ found significant histological alterations of tongue papillae in diabetic rats. Moreover, our LM results of the diabetic group revealed loss of filiform papillae with hyperkeratosis. Spravchikov et al ${ }^{(39)}$ stated that DM causes increased gene expression of keratin associated proteins and keratin complexes in rats which my result in hyperkeratosis.

Moreover, in our study, alterations in taste buds of diabetic group were observed by light microscopy in form of separation between taste bud cells. Also the scanning electron microscopic results showed that some taste pores were ill defined. Many previous studies proved a correlation between diabetes and taste impairment..$^{(9,40-43)}$ The actual mechanism of taste alterations in diabetic patients is still obscure, however, different mechanisms were proposed. Neuropathy which affects the taste nerve's function and microangiopathy affecting taste buds may be important causes of taste impairment in diabetic patients. In the newly diagnosed diabetic patients without complications, the cause may be a defect in the taste receptors. ${ }^{(9,42)}$

Many lines of evidence indicate that all mechanisms of tissue damage associated with hyperglycemia are activated by a single event which is mitochondrial overproduction of ROS.$^{(5)}$ Hyperglycaemia accelerates free radical formation by two mechanisms: rising the inner mitochondrial membrane potential through the generation of excessive free unbound electrons in the respiratory cycle and ROS generation via lipid peroxidation. ${ }^{(44)}$

ROS evoke oxidative stress and can destroy different organelles and bio-molecules. They act as signaling molecules throughout the entire cell death 
pathway involving many biological processes, such as apoptosis, necrosis and autophagy leading to cellular damage and late complications. ${ }^{(45)}$

One of the proposed mechanisms of diabetes related complications is related to the activation of cellular apoptosis..$^{(46,47)}$ The cellular changes which occur during apoptosis are orchestrated by a cascade of proteases called caspases. ${ }^{(48,49)}$ The name caspase was given to these enzymes to indicate the Cysteine requiring ASPartate proteASE activity. Caspase 3 is one of the effector caspases whose activity lead to the characteristic morphological features of apoptosis like cytoplasmic condensation and fragmentation of DNA..$^{(50)}$

Immunohistochemical results of the current study showed intense reaction for caspase 3 in diabetic group 2 in the epithelium covering of the filiform papillae. There was a statistically significant increase in both percentage of surface area of cells with positive reaction to caspase 3 and the optical density of the immunostaining compared to control group. These findings corroborate those of Veluthakal et al (51) who found that diabetes can activate caspase 3 leading to degeneration of rodents' islet cells and subsequent functional defects. Moreover, previous studies proved that hyperglycemia led to upregulation of caspase 3 expression leading to apoptosis of liver ${ }^{(47)}$ and spleen ${ }^{(52)}$ of rats with induced diabetes.

In our study, Quercetin was administrated orally to group 3 at a dose of $50 \mathrm{mg} / \mathrm{kg} /$ day. Previous studies used different doses of Quercetin. Liang et al (53) used 3 different doses of Quercetin 5, 30 \& $50 \mathrm{mg} / \mathrm{kg} /$ day to prevent bone loss in diabetic rats. They found that $5 \mathrm{mg} / \mathrm{kg} / \mathrm{day}$ of Quercetin showed little effect, while higher doses $(30 \& 50 \mathrm{mg} / \mathrm{kg}$ / day) were more effective in prevention of diabetic osteopenia. Moreover, Peng et al ${ }^{(25)}$ concluded that Quercetin at a dose of $50 \mathrm{mg} / \mathrm{kg} /$ day was effective to improve biochemical parameters of glucose and lipids in diabetic rats than at a lower dose of $10 \mathrm{mg} /$ $\mathrm{kg}$ /day. In addition, Mahesh \& Menon (54) found that Quercetin at a dose of $50 \mathrm{mg} / \mathrm{kg} /$ day was more effective in decreasing oxidative stress in diabetic rats than at a higher dose of $80 \mathrm{mg} / \mathrm{kg} /$ day. However, others used a higher dose of $100 \mathrm{mg} / \mathrm{kg} / \mathrm{day}$ and found that it was effective in treatment of rats with induced osteoporosis. ${ }^{(55,56)}$

Examination of the Quercetin-treated group in the present work showed marked improvement in the structure of the tongue papillae compared to diabetic group. Most of the filiform and fungiform papillae appeared with almost its typical shape. These results are in agreement with those of Adewole et al ${ }^{(57)}$ who concluded that Quercetin treatment can reverse the histological changes in pancreatic $\beta$ cells of pancreas in rats due to streptozotocin induced diabetes. This effect was due to the stimulatory effect of quercetin on the endogenous production of antioxidant enzymes.

Quercetin also has a positive effect on oral epithelial cells proliferation. Hujiahemaiti et al ${ }^{(58)}$ stated that Quercetin can stimulate human oral keratinocytes proliferation and enhance reepithelization of oral mucosa in vitro through upregulation of integrin- $\alpha 6 \beta 4$ expression and mRNA and protein levels of transforming growth factor beta 3 (TGF- 33 ) and downregulation of proinflammatory cytokines.

Moreover, previous studies proved that Quercetin is effective in treatment or protection of different cells against different chemical insults. Selvakumar et al ${ }^{(59)}$ and Ansar et al ${ }^{(60)}$ investigated the role of Quercetin in treatment of chemically induced liver damage and found that it can restore to a great degree the normal hepatocyte structure and sinusoid spaces. In addition, Lin et al ${ }^{(61)}$ found that Quercetin can protect rat proximal tubular cells in cell culture against cadmium toxicity.

Scanning electron microscopic results of Quercetin treated group in our study confirmed the light microscopic results where most of the filiform and fungiform papillae appeared almost normal and comparable to those of the control group. 
The ability of Quercetin to overcome the negative effects of diabetes on tissues is mainly through its regulatory effect on blood sugar level and its antioxidant effect. ${ }^{(23)}$ The hypoglycemic effect occurs through the regeneration of pancreatic $\beta$-cells, increase in the level of serum insulin and enhancement of insulin release. Moreover, Quercetin exerts a significant anti-diabetic activity by improving insulin sensitivity, decreasing insulin resistance, and promoting glycogen synthesis. ${ }^{(62)}$ Henagan et al (63) found that chronic Quercetin intake can enhance mitochondrial function. This is accomplished mainly by controlling adenosine triphosphate (ATP) production via inhibition of ATPase and hexokinase enzyme. ${ }^{(64)}$

The strong antioxidant effect of Quercetin are related structurally to the catechol groups in the $\mathrm{B}$ ring and also the free hydroxyl group $(\mathrm{OH}-)$ in the A ring. ${ }^{(65)}$ This configuration makes it ideal for free radical scavenging and metal chelation. ${ }^{(66,67)}$ Pereira Braga et al ${ }^{(68)}$ found that Quercetin increased the levels of antioxidant enzymes in diabetic rats. In addition, it enhances the activation of the phosphoinositide-3-kinase-protein kinase B/Akt (PI3K$\mathrm{PKB} / \mathrm{Akt}$ ) signalling pathway which can improve the overall functions in diabetic subjects. ${ }^{(23)}$

Moreover, Quercetin can upregulate the antioxidant defence mechanism of the body by increasing the levels of glutathione (GSH). Superoxide dismutase (SOD) enzyme captures oxygen free radicals formed in our body like O2- and coverts it into $\mathrm{H} 2 \mathrm{O} 2$. SOD also decomposes $\mathrm{H} 2 \mathrm{O} 2$ to the nontoxic $\mathrm{H} 2 \mathrm{O}$. GSH is important in this reaction as a hydrogen donor. ${ }^{(69,70)}$

The immunohistochemical results of our study showed that in Quercetin treated group the epithelium covering filiform papillae showed a mild reaction for caspase 3 . Statistical analysis revealed a significant decrease in both the area percentage of cells with positive reaction to caspase 3 and the optical density of the immunostaining compared to the diabetic group. This is in accordance with the results of Ola et al (71) who found that Quercetin decreased caspase 3 activity in retina of diabetic rats. In addition, Tuncdemir et al ${ }^{(72)}$ stated that Quercetin decreased immunostaining of caspase 3 in the kidney of diabetic rats compared to untreated group.

In conclusion, based on these results, we can conclude that Quercetin can overcome most of the detrimental effects of diabetes on tongue' filiform and fungiform papillae through its combined antioxidant and anti-apoptotic effect.

\section{REFERENCES}

1. Mealey BL, Ocampo GL. Diabetes mellitus and periodontal disease. Periodontology 2000. 2007;44(1):127-53.

2. Rathmann W, Giani G. Global prevalence of diabetes: estimates for the year 2000 and projections for 2030. Diabetes care. 2004;27(10):2568-9.

3. Shaw JE, Sicree RA, Zimmet PZ. Global estimates of the prevalence of diabetes for 2010 and 2030. Diabetes research and clinical practice. 2010;87(1):4-14.

4. King H, Aubert RE, Herman WH. Global burden of diabetes, 1995-2025: prevalence, numerical estimates, and projections. Diabetes care. 1998;21(9):1414-31.

5. Giacco F, Brownlee M. Oxidative stress and diabetic complications. Circulation research. 2010;107(9):1058-70.

6. Norhammar A, Tenerz A, Nilsson G, Hamsten A, Efendíc $\mathrm{S}$, Rydén $\mathrm{L}$, et al. Glucose metabolism in patients with acute myocardial infarction and no previous diagnosis of diabetes mellitus: a prospective study. The Lancet. 2002;359(9324):2140-4.

7. HE T, CHENG K, GUO X-g. Effects of diabetes mellitus on formation of coronary collateral circulation. Clinical Focus. 2003;23:003.

8. Indurkar MS, Maurya AS, Indurkar S. Oral manifestations of diabetes. Clinical Diabetes. 2016;34(1):54-7.

9. Bhandare NN, Keny MS, Nevrekar RP, Bhandare PN. Diabetic tongue-Could it be a diagnostic criterion? Journal of family medicine and primary care. 2014;3(3):290.

10. Boyce JM, Shone G. Effects of ageing on smell and taste. Postgraduate medical journal. 2006;82(966):239-41.

11. Uddin S, Ahmad S. Dietary antioxidants protection against oxidative stress. Biochemical Education. 1995;23(1):2-7. 
12. Bentz AB. A Review of Quercetin: Chemistry, Antioxident Properties, and Bioavailability. Journal of young investigators. 2017.

13. Ray PD, Huang B-W, Tsuji Y. Reactive oxygen species (ROS) homeostasis and redox regulation in cellular signaling. Cellular signalling. 2012;24(5):981-90.

14. Zaplatic E, Bule M, Shah SZA, Uddin MS, Niaz K. Molecular mechanisms underlying protective role of quercetin in attenuating Alzheimer's disease. Life sciences. 2019.

15. Maritim A, Sanders a, Watkins Iii J. Diabetes, oxidative stress, and antioxidants: a review. Journal of biochemical and molecular toxicology. 2003;17(1):24-38.

16. Asmat U, Abad K, Ismail K. Diabetes mellitus and oxidative stress - a concise review. Saudi Pharmaceutical Journal. 2016;24(5):547-53.

17. Boots AW, Haenen GR, Bast A. Health effects of quercetin: from antioxidant to nutraceutical. European journal of pharmacology. 2008;585(2-3):325-37.

18. Vinayagam R, Xu B. Antidiabetic properties of dietary flavonoids: a cellular mechanism review. Nutrition \& metabolism. 2015;12(1):60.

19. Coskun O, Kanter M, Korkmaz A, Oter S. Quercetin, a flavonoid antioxidant, prevents and protects streptozotocininduced oxidative stress and $\beta$-cell damage in rat pancreas. Pharmacological research. 2005;51(2):117-23.

20. Kwon O, Eck P, Chen S, Corpe CP, Lee J-H, Kruhlak M, et al. Inhibition of the intestinal glucose transporter GLUT2 by flavonoids. The FASEB Journal. 2007;21(2):366-77.

21. Eid HM, Nachar A, Thong F, Sweeney G, Haddad PS. The molecular basis of the antidiabetic action of quercetin in cultured skeletal muscle cells and hepatocytes. Pharmacognosy magazine. 2015;11(41):74

22. Panchal SK, Poudyal H, Brown L. Quercetin ameliorates cardiovascular, hepatic, and metabolic changes in dietinduced metabolic syndrome in rats. The Journal of nutrition. 2012;142(6):1026-32.

23. Shi G-J, Li Y, Cao Q-H, Wu H-X, Tang X-Y, Gao X-H, et al. In vitro and in vivo evidence that quercetin protects against diabetes and its complications: A systematic review of the literature. Biomedicine \& Pharmacotherapy. 2019;109:1085-99.

24. Mahmoud MF, Hassan NA, El Bassossy HM, Fahmy A. Quercetin protects against diabetes-induced exaggerated vasoconstriction in rats: effect on low grade inflammation. PloS one. 2013;8(5):e63784.

25. Peng J, Li Q, Li K, Zhu L, Lin X, Lin X, et al. Quercetin improves glucose and lipid metabolism of diabetic rats: involvement of Akt signaling and SIRT1. Journal of diabetes research. 2017;2017.

26. Feldman AT, Wolfe D. Tissue processing and hematoxylin and eosin staining. Histopathology: Springer; 2014. p. 3143.

27. El-Koshairy N, Hassan RM, Halawa AM. The effect of lead toxicity on albino rats' filiform and fungiform papillae and the possible protective role of honey and black seed. $\mathrm{J}$ Environ Occup Sci-Apr-Jun. 2014;3(2):87.

28. Zedan W, Mourad MI, El-Aziz SMA, Elsabaa HM, Abou Elkhier M. Evaluation of Caspase 3 as a Target for Apoptosis induced via Chemotherapy in Rats. International Journal of Advanced Research. 2015;3:1591-601.

29. Mustafa HN, El Awdan SA, Hegazy GA, Jaleel GAA. Prophylactic role of coenzyme Q10 and Cynara scolymus L on doxorubicin-induced toxicity in rats: Biochemical and immunohistochemical study. Indian journal of pharmacology. 2015;47(6):649.

30. Varghese F, Bukhari AB, Malhotra R, De A. IHC Profiler: an open source plugin for the quantitative evaluation and automated scoring of immunohistochemistry images of human tissue samples. PloS one. 2014;9(5):e96801.

31. Ndisang JF, Vannacci A, Rastogi S. Insulin resistance, type 1 and type 2 diabetes, and related complications 2017. Journal of diabetes research. 2017;2017.

32. Ozgen S, Kilinc OK, Selamoğlu Z. Antioxidant activity of quercetin: a mechanistic review. Turkish Journal of Agriculture-Food Science and Technology. 2016;4(12):1134-8.

33. Lenzen $\mathrm{S}$. The mechanisms of alloxan-and streptozotocininduced diabetes. Diabetologia. 2008;51(2):216-26.

34. Suzuki K, Miyakoshi N, Tsuchida T, Kasukawa Y, Sato K, Itoi E. Effects of combined treatment of insulin and human parathyroid hormone (1-34) on cancellous bone mass and structure in streptozotocin-induced diabetic rats. Bone. 2003;33(1):108-14.

35. Islas-Andrade $\mathrm{S}$, Monsalve MCR, de la Peña JE, Polanco AC, Palomino MA, Velasco AF. Streptozotocin and alloxan in experimental diabetes: comparison of the two models in rats. Acta Histochemica et Cytochemica. 2000; 33(3):201-8. 
36. Akiya O, Serizawa N, Sugihara M, Katagiri S, Kawashima Y. A histological study of changes in the lingual papillae of streptozotocin-induced diabetic rats. The Bulletin of Tokyo Dental College. 1992;33(1):13-24.

37. Mohsen ROM, Halawa AM, Hassan R. Role of bone marrow-derived stem cells versus insulin on filiform and fungiform papillae of diabetic albino rats (light, fluorescent and scanning electron microscopic study). Acta histochemica. 2019;121(7):812-22.

38. Ortug G, Ignak S, Ortug A. Characteristics of lingual papillae in diabetic rats. Morphologie. 2018;102(339):250-4.

39. Spravchikov N, Sizyakov G, Gartsbein M, Accili D, Tennenbaum T, Wertheimer E. Glucose effects on skin keratinocytes: implications for diabetes skin complications. Diabetes. 2001;50(7):1627-35.

40. Bustos-Saldaña R, Alfaro-Rodríguez M, de la Luz SolísRuiz M, Trujillo-Hernández B, Pacheco-Carrasco M, Vázquez-Jiménez C. Taste sensitivity diminution in hyperglycemic type 2 diabetics patients. Revista Médica del Instituto Mexicano del Seguro Social. 2009;47(5):483-8.

41. Bajaj S, Prasad S, Gupta A, Singh VB. Oral manifestations in type-2 diabetes and related complications. Indian journal of endocrinology and metabolism. 2012;16(5):777.

42. Perros P, MacFarlane TW, Counsell C, Frier BM. Altered taste sensation in newly-diagnosed NIDDM. Diabetes care. 1996;19(7):768-70.

43. Wasalathanthri S, Hettiarachchi P, Prathapan S. Sweet taste sensitivity in pre-diabetics, diabetics and normoglycemic controls: a comparative cross sectional study. BMC endocrine disorders. 2014;14(1):67.

44. Ahmad W, Ijaz B, Shabbiri K, Ahmed F, Rehman S. Oxidative toxicity in diabetes and Alzheimer's disease: mechanisms behind ROS/RNS generation. Journal of biomedical science. 2017;24(1):76.

45. He L, He T, Farrar S, Ji L, Liu T, Ma X. Antioxidants maintain cellular redox homeostasis by elimination of reactive oxygen species. Cellular Physiology and Biochemistry. 2017;44(2):532-53.

46. Sadeghi A, Hami J, Razavi S, Esfandiary E, Hejazi Z. The effect of diabetes mellitus on apoptosis in hippocampus: cellular and molecular aspects. International journal of preventive medicine. 2016;7.

47. Francés DE, Ronco MT, Monti JA, Ingaramo PI, Pisani GB, Parody JP, et al. Hyperglycemia induces apoptosis in rat liver through the increase of hydroxyl radical: new insights into the insulin effect. Journal of Endocrinology. 2010;205(2):187.

48. Elmore S. Apoptosis: a review of programmed cell death. Toxicologic pathology. 2007;35(4):495-516.

49. Verhagen AM, Vaux DL. Molecular mechanisms of apoptosis: an overview. Apoptosis: Biology and Mechanisms: Springer; 1999. p. 11-24.

50. Hui H, Dotta F, Mario UD, Perfetti R. Role of caspases in the regulation of apoptotic pancreatic islet beta-cells death. Journal of cellular physiology. 2004;200(2):177-200.

51. Veluthakal R, Arora DK, Goalstone ML, Kowluru RA, Kowluru A. Metabolic stress induces Caspase-3 mediated degradation and inactivation of farnesyl and geranylgeranyl transferase activities in pancreatic $\beta$-cells. Cellular Physiology and Biochemistry. 2016;39(6):2110-20.

52. Hashish HA, Kamal RN. Effect of curcumin on the expression of Caspase- 3 and Bcl-2 in the spleen of diabetic rats. Journal of Experimental and Clinical Anatomy. 2015;14(1):18.

53. Liang W, Luo Z, Ge S, Li M, Du J, Yang M, et al. Oral administration of quercetin inhibits bone loss in rat model of diabetic osteopenia. European journal of pharmacology. 2011;670(1):317-24.

54. Mahesh T, Menon VP. Quercetin allievates oxidative stress in streptozotocin-induced diabetic rats. Phytotherapy Research: An International Journal Devoted to Pharmacological and Toxicological Evaluation of Natural Product Derivatives. 2004;18(2):123-7.

55. Oršolić N, Goluža E, Đikić D, Lisičić D, Sašilo K, Rođak $\mathrm{E}$, et al. Role of flavonoids on oxidative stress and mineral contents in the retinoic acid-induced bone loss model of rat. European journal of nutrition. 2014;53(5):1217-27.

56. Oršolić N, Jeleč Ž, Nemrava J, Balta V, Gregorović G, Jeleč D. Effect of quercetin on bone mineral status and markers of bone turnover in retinoic acid-induced osteoporosis. Polish journal of food and nutrition sciences. 2018;68(2):149-62.

57. Adewole SO, Caxton-Martins EA, Ojewole JA. Protective effect of quercetin on the morphology of pancreatic $\beta$-cells of streptozotocin-treated diabetic rats. African Journal of Traditional, Complementary and Alternative Medicines. 2007;4(1):64-74. 
58. Hujiahemaiti M, Sun X, Zhou J, Lv H, Li X, Qi M, et al. Effects of quercetin on human oral keratinocytes during re-epithelialization: An in vitro study. Archives of oral biology. 2018;95:187-94.

59. Selvakumar K, Bavithra S, Suganya S, Ahmad Bhat F, Krishnamoorthy G, Arunakaran J. Effect of quercetin on haematobiochemical and histological changes in the liver of polychlorined biphenyls-induced adult male wistar rats. Journal of biomarkers. 2013;2013.

60. Ansar S, Siddiqi NJ, Zargar S, Ganaie MA, Abudawood M. Hepatoprotective effect of Quercetin supplementation against Acrylamide-induced DNA damage in wistar rats. BMC complementary and alternative medicine. 2016;16(1):327.

61. Lin W, Lin SQ, HE YL, Gang L, Wang ZY. Protective effects of quercetin on cadmium-induced cytotoxicity in primary cultures of rat proximal tubular cells. Biomedical and Environmental Sciences. 2013;26(4):258-67.

62. Gaballah HH, Zakaria SS, Mwafy SE, Tahoon NM, Ebeid AM. Mechanistic insights into the effects of quercetin and/ or GLP-1 analogue liraglutide on high-fat diet/streptozotocin-induced type 2 diabetes in rats. Biomedicine \& Pharmacotherapy. 2017;92:331-9.

63. Henagan T, Cefalu W, Ribnicky D, Noland R, Dunville $\mathrm{K}$, Campbell W, et al. In vivo effects of dietary quercetin and quercetin-rich red onion extract on skeletal muscle mitochondria, metabolism, and insulin sensitivity. Genes \& nutrition. 2015;10(1):2.

64. de Oliveira MR, Nabavi SM, Braidy N, Setzer WN, Ahmed T, Nabavi SF. Quercetin and the mitochondria: a mechanistic view. Biotechnology advances. 2016;34(5):532-49.

65. Xiao L, Luo G, Tang Y, Yao P. Quercetin and iron metabo- lism: What we know and what we need to know. Food and chemical toxicology. 2018;114:190-203.

66. Heijnen CG, Haenen GR, Minou Oostveen R, Stalpers EM, Bast A. Protection of flavonoids against lipid peroxidation: the structure activity relationship revisited. Free Radical Research. 2002;36(5):575-81.

67. Bule M, Abdurahman A, Nikfar S, Abdollahi M, Amini M. Antidiabetic effect of quercetin: A systematic review and meta-analysis of animal studies. Food and chemical toxicology. 2019.

68. Pereira Braga C, Momentti AC, Barbosa Peixoto F, de Fátima Ferreira Baptista R, dos Santos FA, Fava FH, et al. Influence of treatment with quercetin on lipid parameters and oxidative stress of pregnant diabetic rats. Canadian journal of physiology and pharmacology. 2012; 91(2):171-7.

69. Kobori M, Takahashi Y, Akimoto Y, Sakurai M, Matsunaga I, Nishimuro H, et al. Chronic high intake of quercetin reduces oxidative stress and induces expression of the antioxidant enzymes in the liver and visceral adipose tissues in mice. Journal of Functional Foods. 2015;15:551-60.

70. Granado-Serrano AB, Martín MA, Bravo L, Goya L, Ramos S. Quercetin modulates Nrf2 and glutathione-related defenses in HepG2 cells: Involvement of p38. Chemicobiological interactions. 2012;195(2):154-64.

71. Ola MS, Ahmed M, Shams S, Al-Rejaie SS. Neuroprotective effects of quercetin in diabetic rat retina. Saudi journal of biological sciences. 2017;24(6):1186-94.

72. Tunçdemir M, Mirzataş EB, Uzun H. Renoprotective potential of quercetin in experimental diabetic nephropathy: assesing antiapoptotic and antioxidant effects. Archives of Clinical and Experimental Medicine. 2018;3(3):179-85. 